\title{
Efficient Dipole Parameter Estimation in EEG Systems With Near-ML Performance
}

\author{
Shun Chi Wu*, Student Member, IEEE, A. Lee Swindlehurst, Fellow, IEEE, Po T. Wang, Student Member, IEEE, \\ and Zoran Nenadic, Member, IEEE
}

\begin{abstract}
Source signals that have strong temporal correlation can pose a challenge for high-resolution EEG source localization algorithms. In this paper, we present two methods that are able to accurately locate highly correlated sources in situations where other high-resolution methods such as multiple signal classification and linearly constrained minimum variance beamforming fail. These methods are based on approximations to the optimal maximum likelihood (ML) approach, but offer significant computational advantages over ML when estimates of the equivalent EEG dipole orientation and moment are required in addition to the source location. The first method uses a two-stage approach in which localization is performed assuming an unstructured dipole moment model, and then the dipole orientation is obtained by using these estimates in a second step. The second method is based on the use of the noise subspace fitting concept, and has been shown to provide performance that is asymptotically equivalent to the direct ML method. Both techniques lead to a considerably simpler optimization than ML since the estimation of the source locations and dipole moments is decoupled. Examples using data from simulations and auditory experiments are presented to illustrate the performance of the algorithms.
\end{abstract}

Index Terms-Electroencephalography (EEG), magnetoencephalography (MEG), sensor array processing, source localization.

\section{INTRODUCTION}

$\mathbf{L}$ OCATING sources of cerebral activity using hightemporal-resolution electroencephalography (EEG) and magnetoencephalography (MEG) continues to be an active area of research in neurological science [1]-[4]. Although in general EEG/MEG techniques are reported to have limited spatial resolution, high-resolution techniques such as multiple signal classification (MUSIC) [5], [6] and linearly constrained minimum variance (LCMV) beamforming [7] can be used to cope with this issue. Under certain assumptions, these techniques can resolve very closely spaced sources with high accuracy. To obtain high

Manuscript received October 14, 2011; revised December 30, 2011 and January 18, 2012; accepted January 18, 2012. Date of publication February 10, 2012; date of current version April 20, 2012. This work was supported in part by the National Science Foundation under Grant 1056105 . Asterisk indicates corresponding author.

${ }^{*}$ S. C. Wu is with the Department of Electrical Engineering and Computer Science, University of California, Irvine, CA 92697 USA (e-mail: scwu@ uci.edu).

A. L. Swindlehurst is with the Department of Electrical Engineering and Computer Science, University of California, Irvine, CA 92697 USA (e-mail: swindle@uci.edu).

P. T. Wang and Z. Nenadic are with the Department of Biomedical Engineering, University of California, Irvine, CA 92697 USA (e-mail: ptwang @ uci.edu; znenadic@uci.edu).

Color versions of one or more of the figures in this paper are available online at http://ieeexplore.ieee.org.

Digital Object Identifier 10.1109/TBME.2012.2187336 spatial precision, these techniques normally require a large array of sensors or electrodes for taking EEG/MEG measurements that leads to a complicated high-dimensional inverse problem with an infinite number of solutions. Thus, constraints must be incorporated into the problem for an appropriate solution to exist. In practice, a "forward" propagation model for the brain, skull, and scalp is adopted, and then one attempts to estimate the parameters of the model corresponding to the source activity. One of the most commonly used forward models assumes that the signal source in a small region of the brain originates from an equivalent current dipole, and treats the dipole location and the orientation and magnitude of the dipole moment as parameters to be estimated [8], [9].

While approaches based on MUSIC, LCMV, and their variants have been proposed for EEG localization, their performance degrades significantly if the source signals are highly correlated in time [3], [10], [11]. In such cases, the dimension of the signal subspace collapses, and the subspace is no longer spanned by the lead-field vectors (LFV) of the sources, but rather by some lower dimension linear combination. If prior information about the correlated source locations is available and regions containing these sources can be defined, a beamformer can be constructed to locate and reconstruct the source in only one specified region while rejecting the contribution of sources in other regions [11]. A similar idea is also used in the nulling beamformer of [3], where nulling constraints (i.e., zero gain conditions) are added to cancel signals from specific cortical locations. Unlike the aforementioned beamformers, in which correlated sources are suppressed except for the one to be reconstructed, the dualcore beamformer [4] can simultaneously locate and reconstruct correlated sources using a spatial filter that contains the LFV of two dipole sources at a time. Variants of MUSIC such as recursively applied and projected MUSIC (RAP-MUSIC) [12] and first principal vectors (FINES) [13] have also been shown to have some resilience to highly correlated sources. FINES relies on partitioning the brain into smaller volumes that are isolated via projections, and sources within each volume are determined separately. If two correlated sources are in different subvolumes, then the effect of the correlation can be mitigated through the projection operation. RAP-MUSIC is a sequential technique based on principal angles between subspaces, and has been shown to be effective for highly correlated sources when the number of available data samples is large.

Since both FINES and RAP-MUSIC require a computational load beyond that of standard MUSIC and LCMV, researchers have been led to study the performance of statistically optimal methods such as maximum likelihood (ML) [14]-[17]. While ML techniques do not have difficulty with highly correlated 
sources, they are known to be complex to implement. In particular, unlike MUSIC or LCMV, a direct ML implementation does not allow one to decouple estimation of the linear parameters (dipole orientation and moment magnitude) from that of the source locations. Thus, a complex multidimensional nonlinear optimization over both the location and orientation parameters is unavoidable. Efficient implementations of ML estimate subsets of the parameters (i.e., moment magnitudes, dipole orientations, or positions) by fixing the others as constants, and then alternating through the subsets several times [17]. While this reduces the dimension of the ML search at any given step, the search must be repeated multiple times before convergence. If only the location of the dipole is needed, one can reparameterize the problem by absorbing the dipole orientation and moment together in an unstructured matrix that is estimated directly, and only a one-time search over the locations is required [14], [18]. In one of the approaches presented in this paper, we show how to use these unstructured ML (UML) location estimates to obtain accurate dipole orientation and moment estimates in a second step. We will refer to this method as "decoupled" ML (DML) in the sequel.

As another computationally efficient alternative to MUSIC and LCMV for highly correlated source scenarios, we also propose application of the so-called noise subspace fitting (NSF) algorithm for localization and estimation of the dipole parameters, an algorithm previously developed for direction finding using polarization diversity [19]. The NSF algorithm can be viewed as a multidimensional generalization of MUSIC, and has been shown to asymptotically have the same performance as ML. The form of the NSF cost function allows the dipole location and orientation parameters to be solved for separately, and in fact the solution for the dipole orientation is obtained via a closed-form equation that is more direct and simpler to implement than DML. Both DML and NSF share the following two key advantages: 1) unlike MUSIC and LCMV and their derivatives, they are able to provide accurate estimates in the presence of highly correlated sources, 2) unlike ML, the dimension of the search space is reduced by a factor of 2 since estimation of the dipole orientation and moment is decoupled from that of the location parameters. The performance of the DML and NSF methods will be demonstrated using both simulations and real data from several auditory experiments. While NSF is somewhat simpler to implement than DML, we will see that DML enjoys a slight performance advantage.

In the next section, we present the data model and assumptions made in this study. Section III reviews the standard localization techniques based on MUSIC, LCMV, and ML. The proposed DML and NSF algorithms are then discussed in Sections IV and $\mathrm{V}$, respectively. Simulation results and real-data experiments are described in Sections VI and VII, respectively. Finally, some conclusions are offered in Section VIII.

\section{STANDARD DATA MODEL AND ASSUMPTIONS}

\section{A. Current Dipole Data Model}

Following the mathematical model of [5], [7], the outputs of $m$ sensors sampled at some time instant $t$ are stacked in an $m \times 1$ vector $\mathbf{x}(t)$. The sensor array can be composed of either electrodes as with EEG devices, detector coils as in MEG systems, or both. In this paper, we will focus on the EEG application, although from a mathematical standpoint, the techniques we develop are applicable to MEG localization as well. If $N_{S}$ dipoles are present at time $t$, the output of the array may be modeled as a linear superposition of the signals from each source:

$$
\begin{aligned}
\mathbf{x}(t) & =\mathbf{A} \mathbf{s}(t)+\mathbf{n}(t) \\
& =\mathbf{A} \mathbf{s}(t)+\mathbf{A}_{I} \mathbf{s}_{I}(t)+\mathbf{w}(t)
\end{aligned}
$$

where the $N_{S} \times 1$ signal vector $\mathbf{s}(t)$ is composed of the moment amplitudes of the $N_{S}$ source dipoles, $\mathbf{n}(t) \in \mathbb{R}^{m \times 1}$ is composed of both background electronic/sensor noise $\mathbf{w}(t) \in$ $\mathbb{R}^{m \times 1}$, and contributions from interfering EEG sources that are nonstimulus-related and, therefore, not of interest, $\mathbf{A}_{I} \mathbf{s}_{I}(t) . N_{I}$ represents the number of interfering dipoles and $\mathbf{s}_{I}(t) \in \mathbb{R}^{N_{I} \times 1}$ contains the moment magnitudes of the $N_{I}$ interfering dipoles. The matrix $\mathbf{A}=\left[\mathbf{a}_{1}, \mathbf{a}_{2}, \ldots, \mathbf{a}_{N_{S}}\right]$ is comprised of $N_{S}$ vectors of dimension $m \times 1$ that correspond to the response of the array to each source dipole. These response vectors are often referred to as LFV. Similarly, $\mathbf{A}_{I}$ contains the LFVs of the $N_{I}$ interfering sources. The $k$ th LFV $\mathbf{a}_{k}\left(\mathbf{r}_{k}, \phi_{k}\right)$ represents the response of the sensor array to a unit-amplitude dipole located at position $\mathbf{r}_{k}$ with dipole orientation denoted by

$$
\phi_{k}=\left[\begin{array}{lll}
\phi_{x k} & \phi_{y k} & \phi_{z k}
\end{array}\right]^{T} \text {. }
$$

In practice, a current dipole is a good approximation for a small source viewed from a remote field point [9] and its corresponding LFV can be derived from multilayer spherical head models [8], [20] or based on realistic head measurements taken from computed tomography or magnetic resonance imaging data [21].

The LFV model is typically decomposed into three parts, each due to a separate orientation component:

$$
\begin{aligned}
\mathbf{a}_{k}\left(\mathbf{r}_{k}, \boldsymbol{\phi}_{k}\right) & =\mathbf{G}\left(\mathbf{r}_{k}\right) \boldsymbol{\phi}_{k} \\
& =\left[\mathbf{g}_{x}\left(\mathbf{r}_{k}\right) \mathbf{g}_{y}\left(\mathbf{r}_{k}\right) \mathbf{g}_{z}\left(\mathbf{r}_{k}\right)\right] \boldsymbol{\phi}_{k}
\end{aligned}
$$

for $k=1, \ldots, N_{S}$, where $\mathbf{g}_{x}\left(\mathbf{r}_{k}\right), \mathbf{g}_{y}\left(\mathbf{r}_{k}\right)$, and $\mathbf{g}_{z}\left(\mathbf{r}_{k}\right)$ are the $m \times 1$ LFV's that result from unit amplitude dipole sources oriented in the $x-, y$-, and $z$-directions, respectively. If we stack together the LFVs for the individual direction components of the sources of interest, we can write

$$
\begin{aligned}
& \mathbf{G}_{x}(\mathcal{R})=\left[\mathbf{g}_{x}\left(\mathbf{r}_{1}\right), \ldots, \mathbf{g}_{x}\left(\mathbf{r}_{N_{S}}\right)\right] \in \mathbb{R}^{m \times N_{S}} \\
& \mathbf{G}_{y}(\mathcal{R})=\left[\mathbf{g}_{y}\left(\mathbf{r}_{1}\right), \ldots, \mathbf{g}_{y}\left(\mathbf{r}_{N_{S}}\right)\right] \in \mathbb{R}^{m \times N_{S}} \\
& \mathbf{G}_{z}(\mathcal{R})=\left[\mathbf{g}_{z}\left(\mathbf{r}_{1}\right), \ldots, \mathbf{g}_{z}\left(\mathbf{r}_{N_{S}}\right)\right] \in \mathbb{R}^{m \times N_{S}}
\end{aligned}
$$

and the lead field matrix $\mathbf{A}$ becomes

$$
\begin{aligned}
\mathbf{A}(\mathcal{R}, \boldsymbol{\Phi}) & =\mathbf{G}_{x}(\mathcal{R}) \boldsymbol{\Phi}_{x}+\mathbf{G}_{y}(\mathcal{R}) \boldsymbol{\Phi}_{y}+\mathbf{G}_{z}(\mathcal{R}) \boldsymbol{\Phi}_{z} \\
& =\mathbf{G}(\mathcal{R}) \boldsymbol{\Phi}
\end{aligned}
$$

where

$$
\begin{aligned}
\mathcal{R} & =\left[\mathbf{r}_{1}, \ldots, \mathbf{r}_{N_{S}}\right] \in \mathbb{R}^{3 \times N_{S}} \\
\mathbf{G}(\mathcal{R}) & =\left[\mathbf{G}_{x}(\mathcal{R}) \mathbf{G}_{y}(\mathcal{R}) \mathbf{G}_{z}(\mathcal{R})\right] \in \mathbb{R}^{m \times 3 N_{S}} \\
\boldsymbol{\Phi}_{x} & =\operatorname{diag}\left\{\phi_{x 1}, \ldots, \phi_{x N_{S}}\right\} \in \mathbb{R}^{N_{S} \times N_{S}}
\end{aligned}
$$




$$
\begin{aligned}
\boldsymbol{\Phi}_{y} & =\operatorname{diag}\left\{\phi_{y 1}, \ldots, \phi_{y N_{S}}\right\} \in \mathbb{R}^{N_{S} \times N_{S}} \\
\boldsymbol{\Phi}_{z} & =\operatorname{diag}\left\{\phi_{z 1}, \ldots, \phi_{z N_{S}}\right\} \in \mathbb{R}^{N_{S} \times N_{S}} \\
\boldsymbol{\Phi} & =\left[\boldsymbol{\Phi}_{x} \boldsymbol{\Phi}_{y} \boldsymbol{\Phi}_{z}\right]^{T} \in \mathbb{R}^{3 N_{S} \times N_{S}} .
\end{aligned}
$$

Note also that there is an inherent scaling ambiguity in (1) between each element $s_{k}(t)$ of $\mathbf{s}(t)$ and the corresponding orientation vector $\phi_{k}$. The ambiguity is usually resolved by constraining $\phi_{k}$ in some way, and absorbing any scale factor into $s_{k}(t)$. For example, $\left\|\phi_{k}\right\|=1$ or $\phi_{x k}=1$ are common choices, each with its own implications about the moment amplitude $s_{k}(t)[19]$.

\section{B. Assumptions}

We will adopt the following assumptions regarding the data model described earlier:

A1 The total number of dipole sources (stimulus and nonstimulus related) of sufficient strength to contribute to the model is less than the total number of sensors: $N_{I}+N_{S}<m$.

A2 Any collection of $p \leq m$ LFV forms a linearly independent set (in other words, the sensor array is unambiguous).

A3 The source signals from different dipoles may be highly correlated, but they may not be $100 \%$ correlated with each other (i.e., no dipole signal can be expressed as a linear combination of the other dipole signals).

A4 The location of the $N_{I}$ interfering sources contributing to $\mathbf{n}(t)$ is assumed to be time-invariant during the data collection, but no other assumption is made about their temporal statistics; they need not be white or even stationary. All other components of $\mathbf{n}(t)$ (due to electronic noise, etc.) are assumed to be spatially white with equal variance across all sensors.

Assumptions A2-A4 are standard for existing approaches described in the literature. In most applications of interest, $m$ is large enough and $N_{S}$ is small enough for A1 to be reasonable. Related to A3, the correlation properties of the dipole signals $\mathbf{s}(t)$ are of particular interest in this study. Correlated cortical activities often occur in practice and are thought to be the result of some type of functional connectivity between different regions in the brain [22]. More heuristically, we may expect to see highly correlated sources in EEG signals that are a result of a particular stimulus; these signals are excited simultaneously and often have similar evoked responses. As we will see, the appearance of correlated sources will degrade the localization accuracy of MUSIC and LCMV.

\section{Dual-Condition Experiments and Null Projection}

For EEG measurements, the signals of interest are very weak, and embedded in strong, spatially correlated noise and interference due primarily to background brain activity not related to the stimulus. One approach to cope with this problem is to simultaneously estimate the interference statistics and the noise parameters using the same data, collected over several experiments involving repeated application of the stimulus [16], [17]. If the noise is further assumed to be space-time separable (i.e., the noise covariance matrix is modeled as the Kronecker product of separate spatial and temporal noise covariance matrices), the number of unknown noise parameters to be estimated can be significantly reduced, but the stationarity of the noise in both space and time is still required [17]. Another strategy in such situations is to design experiments with dual conditions, one prior to application of the stimulus (control state) and one after the stimulus has been applied (activity state) [2], [23], [24]. In principle, the control state data will contain only background interference and sensor noise, while the activity state data will contain similar noise and interference as well as the event-related signals:

$$
\begin{aligned}
& \mathbf{x}_{C}(t)=\mathbf{A}_{I} \mathbf{s}_{I C}(t)+\mathbf{w}_{C}(t) \\
& \mathbf{x}_{A}(t)=\mathbf{A} \mathbf{s}(t)+\mathbf{A}_{I} \mathbf{s}_{I A}(t)+\mathbf{w}_{A}(t) .
\end{aligned}
$$

To mitigate interfering EEG sources that are nonstimulusrelated, a null projection (NP) approach has been suggested [24]. In the NP method, a matrix $\mathbf{H} \in \mathbb{R}^{m \times m-N_{I}}$ is found that is as orthogonal as possible to the data collected during the controlstate:

$$
\hat{\mathbf{H}}=\arg \min _{\mathbf{H}}\left\|\mathbf{H}^{T} \mathbf{X}_{C}\right\|_{F}^{2}
$$

where $\mathbf{X}_{C}=\left[\mathbf{x}_{C}(1), \ldots, \mathbf{x}_{C}\left(n_{C}\right)\right]$ represents the control-state data and $n_{C}$ is the number of control state samples. To avoid a trivial solution, the constraint $\mathbf{H}^{T} \mathbf{H}=\mathbf{I}$ is incorporated, and the solution $\hat{\mathbf{H}}$ is the $m-N_{I}$ left singular vectors of $\mathbf{X}_{C}$ corresponding to the smallest singular values. With this matrix computed, the activity state data are then filtered to eliminate the influence of the interference, as follows:

$$
\begin{aligned}
\mathbf{x}_{A}^{\prime}(t) & =\mathbf{P}_{H} \mathbf{x}_{A}(t) \\
& \approx \mathbf{P}_{H} \mathbf{A s}(t)+\mathbf{P}_{H} \mathbf{w}_{A}(t)
\end{aligned}
$$

where $\mathbf{P}_{H}=\hat{\mathbf{H}} \hat{\mathbf{H}}^{T}$. The NP approach has been shown to be superior to techniques based on prewhitening in EEG scenarios where temporal stationarity of the data in the control and activity states cannot be assumed [24].

\section{LOCALIZATION AlgORITHMS}

We briefly review here the MUSIC, LCMV, and ML algorithms for dipole localization incorporating the NP approach for interference suppression. After application of NP, the activity state data will contain the filtered event-related signals and background noise. We define the covariance of the activity-state data as follows:

$$
\begin{aligned}
\hat{\mathbf{R}}_{A}^{\prime} & =\frac{1}{n_{A}} \sum_{t=1}^{n_{A}} \mathbf{x}_{A}^{\prime}(t) \mathbf{x}_{A}^{\prime T}(t) \\
& =\sum_{i=1}^{m} \lambda_{i} \mathbf{e}_{i} \mathbf{e}_{i}^{T}=\mathbf{E}_{S}^{\prime} \boldsymbol{\Lambda}_{S}^{\prime} \mathbf{E}_{S}^{\prime T}+\mathbf{E}_{N}^{\prime} \mathbf{\Lambda}_{N}^{\prime} \mathbf{E}_{N}^{\prime T}
\end{aligned}
$$

where $n_{A}$ is the number of activity state samples, the eigenvalues are ordered as $\lambda_{1} \geq \ldots \geq \lambda_{m}$, the matrix $\mathbf{E}_{S}^{\prime}=\left[\mathbf{e}_{1}, \ldots, \mathbf{e}_{N_{S}}\right]$ contains the eigenvectors corresponding to the $N_{S}$ largest eigenvalues contained in the diagonal matrix $\boldsymbol{\Lambda}_{S}^{\prime}$, and $\mathbf{E}_{N}^{\prime}=$ $\left[\mathbf{e}_{N_{S}+1}, \ldots, \mathbf{e}_{m}\right]$ contain the remaining eigenvectors. We refer to $\mathbf{E}_{S}^{\prime}$ and $\mathbf{E}_{N}^{\prime}$ as the signal and noise subspace eigenvectors, respectively. The value of $N_{S}$ is revealed by the number of 
eigenvalues in $\mathbf{R}_{A}^{\prime}$ that are significantly larger than the variance of the background noise.

\section{A. Multiple Signal Classification}

MUSIC [5], [6], [12] locates the dipoles as those whose LFV's are most orthogonal to $\mathbf{E}_{N}^{\prime}$, i.e.,

$$
\begin{aligned}
\hat{\mathbf{r}}, \hat{\boldsymbol{\phi}} & =\arg \min _{\mathbf{r}, \boldsymbol{\phi}} V_{\mathrm{MU}}(\mathbf{r}, \boldsymbol{\phi}) \\
& =\arg \min _{\mathbf{r}, \boldsymbol{\phi}} \frac{\boldsymbol{\phi}^{T} \mathbf{G}^{\prime T}(\mathbf{r}) \mathbf{E}_{N}^{\prime} \mathbf{E}_{N}^{\prime T} \mathbf{G}^{\prime}(\mathbf{r}) \boldsymbol{\phi}}{\phi^{T} \mathbf{G}^{\prime T}(\mathbf{r}) \mathbf{G}^{\prime}(\mathbf{r}) \boldsymbol{\phi}}
\end{aligned}
$$

where $\mathbf{r}$ is the $3-\mathrm{D}$ coordinates of a possible dipole, $\phi$ is its orientation as defined in (2), and $\mathbf{G}^{\prime}(\mathbf{r})=\mathbf{P}_{H} \mathbf{G}(\mathbf{r})$. A natural constraint for $\phi$ in this case is $\phi^{T} \phi=1$, in which case minimizing the MUSIC criterion becomes equivalent to finding the minima of the following generalized eigenvalue problem:

$$
\begin{aligned}
\hat{\mathbf{r}} & =\arg \min _{\mathbf{r}} \lambda_{\min }(\mathbf{r}) \\
\mathbf{G}^{\prime T}(\hat{\mathbf{r}}) \mathbf{E}_{N}^{\prime} \mathbf{E}_{N}^{\prime T} \mathbf{G}^{\prime}(\hat{\mathbf{r}}) \hat{\boldsymbol{\phi}} & =\lambda_{\min }(\hat{\mathbf{r}}) \mathbf{G}^{\prime T}(\hat{\mathbf{r}}) \mathbf{G}^{\prime}(\hat{\mathbf{r}}) \hat{\boldsymbol{\phi}} .
\end{aligned}
$$

The position estimates are found by viewing $\lambda_{\min }$ as a function of $\mathbf{r}$ and then searching for its minima. The orientation of the dipole with location estimate $\hat{\mathbf{r}}$ is then taken to be the unit-norm eigenvector associated with $\lambda_{\min }(\hat{\mathbf{r}})$. MUSIC is referred to as a "scanning" method, since it relies on a scan of the 3-D volume in order to localize potential sources. Note that MUSIC will fail when any subset of the source signals are highly correlated, since in this case $\mathbf{E}_{N}^{\prime}$ will be orthogonal to some linear combination of the LFV's in a lower dimensional subspace, rather than the LFV's themselves [19].

\section{B. Linearly Constrained Beamforming}

The LCMV approach scans by forming "beams" that minimize power subject to a unit gain constraint at each potential source location [2], [3], [7]. This leads to finding the minima of the following cost function:

$$
\begin{aligned}
\hat{\mathbf{r}}, \hat{\boldsymbol{\phi}} & =\arg \min _{\mathbf{r}, \boldsymbol{\phi}} V_{\mathrm{LCMV}}(\mathbf{r}, \boldsymbol{\phi}) \\
& =\arg \min _{\mathbf{r}, \boldsymbol{\phi}} \frac{\boldsymbol{\phi}^{T} \mathbf{G}^{\prime T}(\mathbf{r})\left(\mathbf{R}_{A}^{\prime}\right)^{-1} \mathbf{G}^{\prime}(\mathbf{r}) \boldsymbol{\phi}}{\boldsymbol{\phi}^{T} \mathbf{G}^{\prime T}(\mathbf{r}) \mathbf{G}^{\prime}(\mathbf{r}) \boldsymbol{\phi}} .
\end{aligned}
$$

The positions and orientations are then found in the same fashion as in MUSIC:

$$
\begin{aligned}
\hat{\mathbf{r}} & =\arg \min _{\mathbf{r}} \lambda_{\min }(\mathbf{r}) \\
\mathbf{G}^{\prime T}(\hat{\mathbf{r}})\left(\mathbf{R}_{A}^{\prime}\right)^{-1} \mathbf{G}^{\prime}(\hat{\mathbf{r}}) \hat{\boldsymbol{\phi}} & =\lambda_{\min }(\hat{\mathbf{r}}) \mathbf{G}^{\prime T}(\hat{\mathbf{r}}) \mathbf{G}^{\prime}(\hat{\mathbf{r}}) \hat{\boldsymbol{\phi}} .
\end{aligned}
$$

The LCMV approach also performs poorly for highly correlated signals due to the fact that the LCMV minimization may cancel the desired signal despite maintaining unit gain at the signal's location.

\section{Maximum Likelihood or Least-Squares Method}

The performance limitations of MUSIC and LCMV have led some to consider ML approaches to the EEG source localization problems. If the noise remaining after prewhitening or NP is modeled as a spatially white Gaussian process, then ML is equivalent to a least-squares fit of the model to the data [14], [15]:

$$
V(\mathcal{R}, \boldsymbol{\Phi})=\left\|\mathbf{X}_{A}^{\prime}-\mathbf{P}_{\mathbf{A}^{\prime}(\mathcal{R}, \Phi)} \mathbf{X}_{A}^{\prime}\right\|_{F}^{2}
$$

where

$$
\begin{aligned}
\mathbf{X}_{A}^{\prime} & =\left[\mathbf{x}_{A}^{\prime}(1), \ldots, \mathbf{x}_{A}^{\prime}\left(n_{A}\right)\right] \in \mathbb{R}^{m \times n_{A}} \\
\mathbf{P}_{\mathbf{A}^{\prime}(\mathcal{R}, \boldsymbol{\Phi})} & =\mathbf{A}^{\prime}(\mathcal{R}, \boldsymbol{\Phi})\left[\mathbf{A}^{\prime T}(\mathcal{R}, \boldsymbol{\Phi}) \mathbf{A}^{\prime}(\mathcal{R}, \boldsymbol{\Phi})\right]^{-1} \mathbf{A}^{\prime T}(\mathcal{R}, \boldsymbol{\Phi}) \\
\mathbf{A}^{\prime}(\mathcal{R}, \boldsymbol{\Phi}) & =\mathbf{P}_{H} \mathbf{A}(\mathcal{R}, \boldsymbol{\Phi})
\end{aligned}
$$

and $\|\cdot\|_{F}$ denotes the Frobenius norm. While the ML method is immune to problems that result from correlated signals, it comes at the expense of increased complexity in the optimization. In particular, the ML approach is not a scanning method, and it is not possible in general to decouple the estimation of $\mathcal{R}$ and $\boldsymbol{\Phi}$, since the $\boldsymbol{\Phi}$ parameters are embedded in the nonlinear inverse of the projection matrix. Instead, one must simultaneously perform a constrained $6 N_{S}$-dimensional nonlinear optimization over $3 N_{S}$ location and $3 N_{S}$ dipole moment parameters. A more efficient implementation is possible by alternately fixing $\boldsymbol{\Phi}, \mathcal{R}$ and the moment magnitudes, and then estimating the others. While the source localization step of this process requires a reduced dimension $\left(3 N_{S}\right)$ search, several iterations of this procedure are required to obtain convergence of the estimates [17].

If one is only concerned with the locations of the dipoles and not their orientation, a possible simplification is to assume an unstructured dipole moment model in which the dipole orientation is not assumed to be constant over the observation interval [14], [18]. In the unstructured dipole model, the signal emitted by a source is no longer modeled as a rank-one signal $\mathbf{G}(\mathbf{r}) \boldsymbol{\phi} s(t)$, where $\mathbf{G}(\mathbf{r})$ is $m \times 3, \boldsymbol{\phi}$ is $3 \times 1$ and $s(t)$ is a scalar. Instead, the signal is written as $\mathbf{G}(\mathbf{r}) \mathbf{s}^{\prime}(t)$ where $\mathbf{s}^{\prime}(t)$ is $3 \times 1$ and is left unparameterized. The unstructured model does not require the dipole moment to be constant; in fact, it can be arbitrary at every time $t$. This assumption may lead to degraded estimation performance when the dipole moment is constant (as we assume it to be), but it results in a simpler ML solution since $\mathbf{s}^{\prime}(t)$ can be estimated very simply, in closed form. The unstructured signal model leads to the following modified ML criterion:

$$
V_{\mathrm{ML}}(\mathcal{R})=\left\|\mathbf{X}_{A}^{\prime}-\mathbf{P}_{\mathbf{G}^{\prime}(\mathcal{R})} \mathbf{X}_{A}^{\prime}\right\|_{F}^{2}
$$

where

$$
\begin{aligned}
\mathbf{P}_{\mathbf{G}^{\prime}(\mathcal{R})} & =\mathbf{G}^{\prime}(\mathcal{R})\left[\mathbf{G}^{\prime T}(\mathcal{R}) \mathbf{G}^{\prime}(\mathcal{R})\right]^{-1} \mathbf{G}^{\prime T}(\mathcal{R}) \\
\mathbf{G}^{\prime}(\mathcal{R}) & =\mathbf{P}_{H} \mathbf{G}(\mathcal{R}) .
\end{aligned}
$$

Minimization of (11) requires only a single $3 N_{S}$-dimensional search, but it can resolve fewer source locations. We will refer to this approach as UML.

\section{Decoupled Maximum LiKelihood}

As mentioned earlier, the performance of MUSIC and LCMV will degrade in the presence of highly correlated sources. While the ML method has no difficulty with correlated (or even coherent) sources provided $N_{S}$ is known, it requires a constrained nonlinear optimization over a large number of parameters, which 
may be prohibitive. In this section, we show how to use the UML location estimates discussed earlier to obtain separate estimates of the dipole orientations and moment amplitudes.

Substituting the location estimates provided by UML into (1) and (3), the activity-state data can be represented as (ignoring noise for the moment)

$$
\begin{aligned}
\mathbf{X}_{A}^{\prime} & =\left[\mathbf{G}^{\prime}\left(\hat{\mathbf{r}}_{1}\right), \ldots, \mathbf{G}^{\prime}\left(\hat{\mathbf{r}}_{N_{S}}\right)\right]\left[\begin{array}{c}
\phi_{1} \mathbf{s}_{1} \\
\vdots \\
\phi_{N_{S}} \mathbf{s}_{N_{S}}
\end{array}\right] \\
& =\hat{\mathbf{G}}^{\prime}\left[\begin{array}{c}
\mathbf{M}_{1} \\
\vdots \\
\mathbf{M}_{N_{S}}
\end{array}\right]=\hat{\mathbf{G}}^{\prime} \mathbf{M}
\end{aligned}
$$

where $\mathbf{s}_{k}=\left[s_{k}(1), \ldots, s_{k}\left(n_{A}\right)\right], \mathbf{M}_{k} \in \mathbb{R}^{3 \times n_{A}}$ is the moment of the dipole located at the $k$ th estimated position $\hat{\mathbf{r}}_{k}$, and $\hat{\mathbf{G}}^{\prime}$ contains the lead field matrices of the sources evaluated at the estimated positions. UML provides an unstructured estimate of the moment matrix $\mathbf{M}$ via

$$
\hat{\mathbf{M}}=\left(\hat{\mathbf{G}}^{\prime T} \hat{\mathbf{G}}^{\prime}\right)^{-1} \hat{\mathbf{G}}^{\prime T} \mathbf{X}_{A}^{\prime}
$$

While the dipole orientations and moment amplitudes are not directly revealed in (13), the DML approach described here illustrates how to determine $\mathbf{s}_{k}$ and $\phi_{k}$ from $\hat{\mathbf{M}}$ in a straightforward way. The $3 N_{S} \times n_{A}$ matrix $\hat{\mathbf{M}}$ can be partitioned into $N_{S}$ blocks of dimension $3 \times n_{A}$ that represent estimates of the moment matrix for each individual source $\mathbf{M}_{k}=\phi_{k} \mathbf{s}_{k}$. Since the product $\phi_{k} \mathbf{s}_{k}$ is rank-one, a straightforward solution to the problem under the constraint $\left\|\phi_{k}\right\|=1$ would be to define the singular value decomposition $\hat{\mathbf{M}}_{k}=\mathbf{U}_{k} \boldsymbol{\Sigma}_{k} \mathbf{V}_{k}^{T}$ and set

$$
\begin{aligned}
\hat{\boldsymbol{\phi}}_{k} & =\mathbf{u}_{k, 1} \\
\hat{\mathbf{s}}_{k} & =\sigma_{k, 1} \mathbf{v}_{k, 1}^{T}
\end{aligned}
$$

where $\mathbf{u}_{k, 1}$ and $\mathbf{v}_{k, 1}$ are the left and right singular vectors of $\hat{\mathbf{M}}_{k}$ corresponding to the largest singular value $\sigma_{k, 1}$, respectively.

The DML algorithm for estimating $\mathcal{R}, \phi_{k}$, and $\mathbf{s}_{k}$ for $k=$ $1, \ldots, N_{S}$, thus, consists of the following two steps:

1) Find estimates of the source locations $\hat{\mathcal{R}}$ using UML with (11).

2) Solve for $\hat{\phi}_{k}$ and $\hat{\mathbf{s}}_{k}$ using $\hat{\mathcal{R}},(13)$ and the singular vectors in (14)-(15).

\section{Noise Subspace FitTing}

As an alternative to the DML algorithm described in the previous section, here we present an approach based on NSF that achieves ML-like performance while reducing the dimension of the search by one half. The NSF approach was first introduced in [25] in the context of direction finding with uniform linear arrays, and later generalized in [19] to arbitrary diversely polarized antenna arrays, which have an array response model similar to that for EEG current dipoles. In [25], it was shown that NSF is a "large sample" realization of ML, with equivalent asymptotic performance. For the application considered in this paper, the NSF algorithm estimates the dipole positions and moments as those that minimize the following cost function:

$$
V_{\mathrm{NSF}}(\mathcal{R}, \boldsymbol{\Phi})=\operatorname{Tr}\left\{\mathbf{A}^{\prime T}(\mathcal{R}, \boldsymbol{\Phi}) \mathbf{E}_{N}^{\prime} \mathbf{E}_{N}^{\prime T} \mathbf{A}^{\prime}(\mathcal{R}, \boldsymbol{\Phi}) \mathbf{W}\right\}
$$

where $\mathbf{E}_{N}^{\prime}$ is defined in (7). For the asymptotic equivalence with ML to hold, the $N_{S} \times N_{S}$ weighting matrix $\mathbf{W}=\mathbf{W}^{T}>0$ must be chosen as

$$
\mathbf{W}=\left[\mathbf{A}^{\prime T}\left(\hat{\mathcal{R}}_{i}, \hat{\mathbf{\Phi}}_{i}\right) \mathbf{E}_{S}^{\prime}\left(\Lambda_{S}^{\prime}-\hat{\sigma}^{2} \mathbf{I}\right)^{-2} \Lambda_{S}^{\prime} \mathbf{E}_{S}^{\prime T} \mathbf{A}^{\prime}\left(\hat{\mathcal{R}}_{i}, \hat{\boldsymbol{\Phi}}_{i}\right)\right]^{-1}
$$

using definitions from (7), where $\hat{\sigma}^{2}$ is an estimate of the residual noise variance in $\mathbf{X}_{A}^{\prime}$ [for example, $\hat{\sigma}^{2}=\operatorname{Tr}\left(\boldsymbol{\Lambda}_{N}^{\prime} /\left(m-N_{S}\right)\right.$ ], and $\hat{\mathcal{R}}_{i}$ and $\hat{\boldsymbol{\Phi}}_{i}$ are initial consistent estimates of the parameters, obtained for example by application of the algorithm with an arbitrary positive definite weighting matrix. The fact that NSF is a multidimensional generalization of MUSIC can be seen from the fact that, when $\mathbf{W}=\mathbf{I}$, the diagonal elements of the argument of the trace operation in (16) represent the MUSIC cost function simultaneously evaluated at $N_{S}$ different source locations.

At first glance, it appears that, like ML, minimizing (16) will lead to a $6 N_{S}$-dimensional nonlinear constrained optimization. However, unlike ML, the form of (16) allows one to decouple the estimation of the dipole locations $\mathcal{R}$ and moments $\boldsymbol{\Phi}$, and leads to a closed-form solution for $\boldsymbol{\Phi}$. The NSF criterion can, thus, be concentrated with respect to $\Phi$, which results in a cost function involving only the $3 N_{S}$ location parameters in $\mathcal{R}$. For the EEG application, searching only over $\mathcal{R}$ is a considerable advantage not only because of the reduced dimension, but also because the search can often be confined to certain regions of interest in the brain where energy is expected to be present.

To illustrate the decoupling mathematically, defining $\mathbf{P}=$ $\mathbf{E}_{N}^{\prime} \mathbf{E}_{N}^{\prime T}$ and substituting (4) for $\mathbf{A}(\mathcal{R}, \boldsymbol{\Phi})$ in (16), we have

$$
\begin{aligned}
& V_{\mathrm{NSF}}(\mathcal{R}, \boldsymbol{\Phi}) \\
& =\operatorname{Tr}\left\{\boldsymbol{\Phi}_{x}^{T} \mathbf{G}_{x}^{\prime T} \mathbf{P} \boldsymbol{\Phi}_{x} \mathbf{G}_{x}^{\prime} \mathbf{W}\right\}+\operatorname{Tr}\left\{\boldsymbol{\Phi}_{x}^{T} \mathbf{G}_{x}^{\prime T} \mathbf{P} \boldsymbol{\Phi}_{y} \mathbf{G}_{y}^{\prime} \mathbf{W}\right\} \\
& \quad+\operatorname{Tr}\left\{\boldsymbol{\Phi}_{x}^{T} \mathbf{G}_{x}^{\prime T} \mathbf{P} \boldsymbol{\Phi}_{z} \mathbf{G}_{z}^{\prime} \mathbf{W}\right\}+\operatorname{Tr}\left\{\boldsymbol{\Phi}_{y}^{T} \mathbf{G}_{y}^{\prime T} \mathbf{P} \boldsymbol{\Phi}_{x} \mathbf{G}_{x}^{\prime} \mathbf{W}\right\} \\
& \quad+\operatorname{Tr}\left\{\boldsymbol{\Phi}_{y}^{T} \mathbf{G}_{y}^{\prime T} \mathbf{P} \boldsymbol{\Phi}_{y} \mathbf{G}_{y}^{\prime} \mathbf{W}\right\}+\operatorname{Tr}\left\{\boldsymbol{\Phi}_{y}^{T} \mathbf{G}_{y}^{\prime T} \mathbf{P} \boldsymbol{\Phi}_{z} \mathbf{G}_{z}^{\prime} \mathbf{W}\right\} \\
& \quad+\operatorname{Tr}\left\{\boldsymbol{\Phi}_{z}^{T} \mathbf{G}_{z}^{\prime T} \mathbf{P} \boldsymbol{\Phi}_{x} \mathbf{G}_{x}^{\prime} \mathbf{W}\right\}+\operatorname{Tr}\left\{\boldsymbol{\Phi}_{z}^{T} \mathbf{G}_{z}^{T} \mathbf{P} \boldsymbol{\Phi}_{y} \mathbf{G}_{y}^{\prime} \mathbf{W}\right\} \\
& \quad+\operatorname{Tr}\left\{\boldsymbol{\Phi}_{z}^{T} \mathbf{G}_{z}^{\prime T} \mathbf{P} \boldsymbol{\Phi}_{z} \mathbf{G}_{z}^{\prime} \mathbf{W}\right\}
\end{aligned}
$$

where $\mathbf{G}_{x}^{\prime}=\mathbf{P}_{H} \mathbf{G}_{x}$ with its argument having been dropped for notational simplicity, and similar definitions hold for $\mathbf{G}_{y}^{\prime}$ and $\mathbf{G}_{z}^{\prime}$. We then use the following result, which holds for any diagonal matrices $\boldsymbol{\Phi}_{x}, \boldsymbol{\Phi}_{y}$ and for arbitrary matrices $\mathbf{X}$ and $\mathbf{Y}$ :

$$
\operatorname{Tr}\left(\boldsymbol{\Phi}_{x}^{T} \mathbf{X} \boldsymbol{\Phi}_{y} \mathbf{Y}\right)=\boldsymbol{\phi}_{x}^{T}(\mathbf{X} \odot \mathbf{Y}) \boldsymbol{\phi}_{y}
$$

where $\odot$ represents a Hadamard/Schur (element by element) product, $\phi_{x}$ is the vector formed from the diagonal elements of $\boldsymbol{\Phi}_{x}$, and a similar definition holds for $\phi_{y}$ (and $\phi_{z}$ ). Using (19), we can rewrite the NSF cost function as

$$
V_{\mathrm{NSF}}\left(\mathcal{R}, \phi_{x y z}\right)=\phi_{x y z}^{T} \mathbf{M}_{S}(\mathcal{R}) \phi_{x y z}
$$


where $\phi_{x y z}=\left[\begin{array}{lll}\phi_{x}^{T} & \phi_{y}^{T} & \phi_{z}^{T}\end{array}\right]^{T}$, and $\mathbf{M}_{S}(\mathcal{R})=$

$$
\left[\begin{array}{ccc}
\left(\mathbf{G}_{x}^{T} \mathbf{P} \mathbf{G}_{x}^{\prime}\right) \odot \mathbf{W} & \left(\mathbf{G}_{x}^{\prime T} \mathbf{P} \mathbf{G}_{y}^{\prime}\right) \odot \mathbf{W} & \left(\mathbf{G}_{x}^{\prime T} \mathbf{P} \mathbf{G}_{z}^{\prime}\right) \odot \mathbf{W} \\
\left(\mathbf{G}_{y}^{\prime T} \mathbf{P G}_{x}^{\prime}\right) \odot \mathbf{W} & \left(\mathbf{G}_{y}^{\prime T} \mathbf{P G}_{y}^{\prime}\right) \odot \mathbf{W} & \left(\mathbf{G}_{y}^{\prime T} \mathbf{P G}_{z}^{\prime}\right) \odot \mathbf{W} \\
\left(\mathbf{G}_{z}^{\prime T} \mathbf{P} \mathbf{G}_{x}^{\prime}\right) \odot \mathbf{W} & \left(\mathbf{G}_{z}^{T} \mathbf{P} \mathbf{G}_{y}^{\prime}\right) \odot \mathbf{W} & \left(\mathbf{G}_{z}^{\prime T} \mathbf{P} \mathbf{G}_{z}^{\prime}\right) \odot \mathbf{W}
\end{array}\right] .
$$

Due to the form of the cost function in (20), in which $\mathbf{M}_{S}(\mathcal{R})$ is only a function of dipole positions $\mathcal{R}$, it is natural to suppose that minimizing $V_{\mathrm{NSF}}$ is equivalent to finding the dipole positions $\mathcal{R}$ that minimize the smallest eigenvalue of $\mathbf{M}_{S}(\mathcal{R})$, and obtaining the dipole orientation parameters from the eigenvector associated with this minimum eigenvalue. However, this is not the case because to guarantee identifiability, a constraint on $\phi_{x y z}$ is required to prevent the orientation vector for a subset of the signals from going to zero [19], which can occur even under the unit norm constraint. Since the orientation components can be scaled arbitrarily, one approach is simply to force $\Phi_{x}=\mathbf{I}_{N_{S} \times N_{S}}$, for example. This constraint implicitly assumes that all of the sources of interest have nonzero orientation in the $x$-direction. If needed, the algorithm can also be run with similar constraints for the $y$ - and $z$-directions to ensure that sources with moments nearly orthogonal to the $x$-axis are isolated. As was discussed in Section II-A, constraining the orientation of individual dipoles to have unit norm (i.e., $\left\|\phi_{x y z}\right\|=1$ ) is an alternative approach to avoiding trivial solutions. However, this approach prevents the decoupling of the estimation of $\Phi$ and $\mathcal{R}$, and negates the advantage of the NSF approach (e.g., one could simply use ML instead if a $6 N_{s}$-dimensional search was required).

Minimizing (20) over $\mathcal{R}$ and $\boldsymbol{\phi}_{y z}=\left[\begin{array}{ll}\boldsymbol{\phi}_{y}^{T} & \boldsymbol{\phi}_{z}^{T}\end{array}\right]^{T}$ with $\boldsymbol{\Phi}_{x}=\mathbf{I}$, we have

$$
\begin{aligned}
\hat{\mathcal{R}}, \hat{\boldsymbol{\phi}}_{y z} & =\arg \min _{\mathcal{R}, \boldsymbol{\phi}_{y z}} \boldsymbol{\psi}^{T}\left[\begin{array}{lll}
\mathbf{M}_{S, x x} & \mathbf{M}_{S, x y} & \mathbf{M}_{S, x z} \\
\mathbf{M}_{S, y x} & \mathbf{M}_{S, y y} & \mathbf{M}_{S, y z} \\
\mathbf{M}_{S, z x} & \mathbf{M}_{S, z y} & \mathbf{M}_{S, z z}
\end{array}\right] \boldsymbol{\psi} \\
= & \arg \min _{\mathcal{R}, \boldsymbol{\phi}_{y z}} \boldsymbol{\psi}^{T}\left[\begin{array}{cc}
\mathbf{M}_{S, x x} & \mathbf{M}_{S 1} \\
\mathbf{M}_{S 3} & \mathbf{M}_{S 2}
\end{array}\right] \boldsymbol{\psi} \\
= & \arg \min _{\mathcal{R}, \boldsymbol{\phi}_{y z}}\left(\mathbf{e}^{T} \mathbf{M}_{S, x x} \mathbf{e}+\mathbf{e}^{T} \mathbf{M}_{S 1} \boldsymbol{\phi}_{y z}\right. \\
& \left.+\boldsymbol{\phi}_{y z}^{T} \mathbf{M}_{S 3} \mathbf{e}+\boldsymbol{\phi}_{y z}^{T} \mathbf{M}_{S 2} \boldsymbol{\phi}_{y z}\right)
\end{aligned}
$$

where $\mathbf{e}$ is a vector composed of $N_{S}$ ones, $\boldsymbol{\psi}=\left[\mathbf{e}^{T} \boldsymbol{\phi}_{y z}^{T}\right]^{T}$, and $\mathbf{M}_{S}(\mathcal{R})$ has been partitioned into blocks in an obvious way. Because the cost function is quadratic in $\phi_{y z}$, the estimation of $\phi_{y z}$ is separable from that of the dipole positions and can be found in closed form. Setting $\partial V_{\mathrm{NSF}} / \partial \phi_{y z}=0$, we have

$$
\hat{\phi}_{y z}=-\frac{1}{2} \mathbf{M}_{S 2}^{-1}\left(\mathbf{M}_{S 1}^{T}+\mathbf{M}_{S 3}\right) \mathbf{e}=\mathbf{M e}
$$

where $\mathbf{M}=-\frac{1}{2} \mathbf{M}_{S 2}^{-1}\left(\mathbf{M}_{S 1}^{T}+\mathbf{M}_{S 3}\right)$. Substituting this expression into (21) leads to a concentrated cost function that depends only on $\mathcal{R}$ :

$V_{\mathrm{NSF}}(\mathcal{R})=\mathbf{e}^{T}\left(\mathbf{M}_{S, x x}+\mathbf{M}_{S 1} \mathbf{M}+\mathbf{M}^{T} \mathbf{M}_{S 3}+\mathbf{M}^{T} \mathbf{M}_{S 2} \mathbf{M}\right) \mathbf{e}$.

The NSF algorithm for estimating $\mathcal{R}$ and $\phi_{y z}$, thus, consists of the following two steps:
1) Find the locations $\mathcal{R}$ that minimize the sum of the elements of

$$
\mathbf{M}_{S, x x}+\mathbf{M}_{S 1} \mathbf{M}+\mathbf{M}^{T} \mathbf{M}_{S 3}+\mathbf{M}^{T} \mathbf{M}_{S 2} \mathbf{M} .
$$

2) Solve for $\phi_{y z}$ using $\hat{\mathcal{R}}$ in (22).

A similar procedure is obtained if one constrains the $y$-or $z$-component of the dipole moments using $\boldsymbol{\Phi}_{y}=\mathbf{I}$ or $\boldsymbol{\Phi}_{z}=\mathbf{I}$ instead of $x$.

\section{SIMULATION EXAMPLES}

\section{A. Simulation Parameters}

As an example, we choose an EEG simulation involving 61 electrodes from the 10-10 system [26]. The LFV's were calculated using the approximate method in [8], in which the head was modeled as a four-layer sphere with its radii and conductivities specified in [27]. The number of data points used in both the control and activity states was set to 100 and 25 interfering sources were simulated at random locations on the surface of brain with random dipole orientations. Rather than generating them synthetically, the waveforms used for the interfering sources were taken from the electrode outputs of real EEG measurements. The moment magnitudes for the sources of interest were chosen to be similar to the averaged evoked response potentials (ERP) illustrated in [28]. White noise was added to both control and activity state data, and it was scaled such that its power matched the desired signal-to-noise ratio (SNR), defined as $\left\|\mathbf{A}_{S} \mathbf{S}_{S}\right\|_{F}^{2} /\left\|\mathbf{W}_{A}\right\|_{F}^{2}$, where $\mathbf{A}_{S}$ represents the LFV's of the sources of interest, $\mathbf{S}_{S}=\left[\mathbf{s}_{S}(1), \ldots, \mathbf{s}_{S}\left(n_{A}\right)\right]$ contains the dipole amplitudes of the sources of interest, and $\mathbf{W}_{A}$ is the spatially white background noise present during the activity states. Each signal of interest was scaled to have unit power and all interference waveforms were simulated with the same power specified by the SIR $\left\|\mathbf{A}_{S} \mathbf{S}_{S}\right\|_{F}^{2} /\left\|\mathbf{A}_{I} \mathbf{S}_{I}\right\|_{F}^{2}$, in which $\mathbf{A}_{I}$ is the LFV's of the interfering sources and $\mathbf{S}_{I}$ contains the dipole amplitudes of the interfering sources.

\section{B. Results and Discussion}

We first compare the performance of MUSIC, LCMV, DML, and NSF using simulated data with two correlated sources whose SNR and SIR were 15 and $0 \mathrm{~dB}$, respectively. Figs. 1 and 2 show the spatial spectra obtained by evaluating the reciprocals of (8) and (9) at the grid points (we plot the inverse of the cost functions so that the peaks in the spectrum approximately correspond to the source locations estimated by MUSIC and LCMV). Dipole location estimates obtained using NSF (white squares) and the DML method (magenta diamonds) are plotted on top of the spectra as well. Black dots show the locations of the interfering sources. For Fig. 1, two dipoles were located on the surface of the brain near sensors C5 and C6 to mimic the auditory experiment described in the next section. Fig. 2 corresponds to a case with two more closely spaced dipole sources near sensors $\mathrm{C} 1$ and $\mathrm{C} 2$. For this figure, the locations of the true sources are indicated by green triangles and only results for MUSIC are presented since the performance of LCMV was very similar. In both cases, the dipole orientation was random and the correlation 


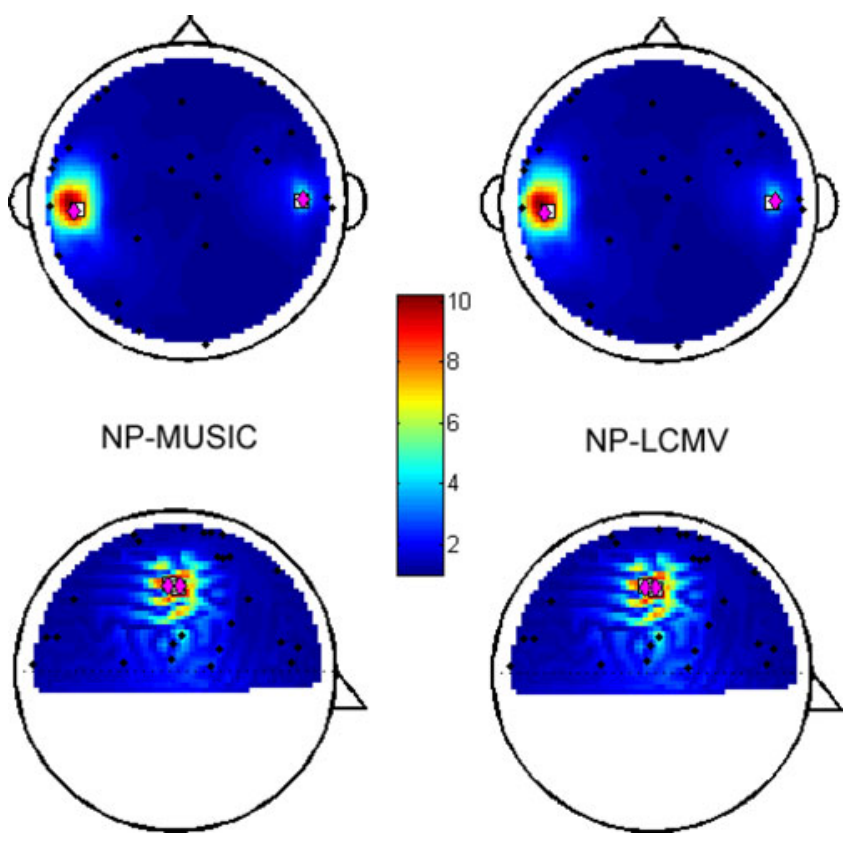

Fig. 1. Spatial spectra and location estimates obtained by the algorithms studied in this paper. Black dots represent locations of interferers, white squares, and magenta diamonds represent position estimates obtained by NSF and DML, respectively. The SIR and SNR were, respectively, 0 and $15 \mathrm{~dB}$ and the correlation coefficient was 0.95 . NP is used to emphasize that the null projection is done before source localization.

coefficient was 0.95 . Signal cancellation effects due to the high correlation [3], [29] cause one of the source peaks in the MUSIC and LCMV spectra to be nearly too faint to be discerned. In the zoomed-in part of Fig. 2, we also see that the location estimates provided by MUSIC for both sources is much less accurate than those from NSF and DML.

To quantify the performance gain of DML and NSF, we conducted a series of Monte Carlo trials in which the two simulated dipoles were placed in fixed positions on the surface of the brain, near sensors $\mathrm{C} 5$ and $\mathrm{C} 6$, with random orientations and various levels of correlation between the two source signals were tested. The SNR and SIR were also 15 and $0 \mathrm{~dB}$, respectively. For comparison, the ML algorithm (10) was also implemented with a full $6 N_{S}$-dimensional search. Figs. 3 and 4 show the RMS location and orientation errors of the algorithms obtained by averaging over the Monte Carlo trials. As shown in the figures, the performance advantage of NSF, ML, and DML over MUSIC and LCMV becomes increasingly greater as the correlation of the signals increases. Note that DML enjoys a slight performance advantage over NSF for both the location and dipole orientation estimates. Moreover, the ML algorithm performs similarly to NSF and DML, but requires significantly more computation.

\section{AUDITORY EXPERIMENTS}

Experiments with auditory stimuli were conducted with a single human subject to elicit auditory-evoked potentials. The study was approved by the University of California Institutional Review Board and the subject signed an informed consent form. The subject received the audio stimulus through a pair of stereo

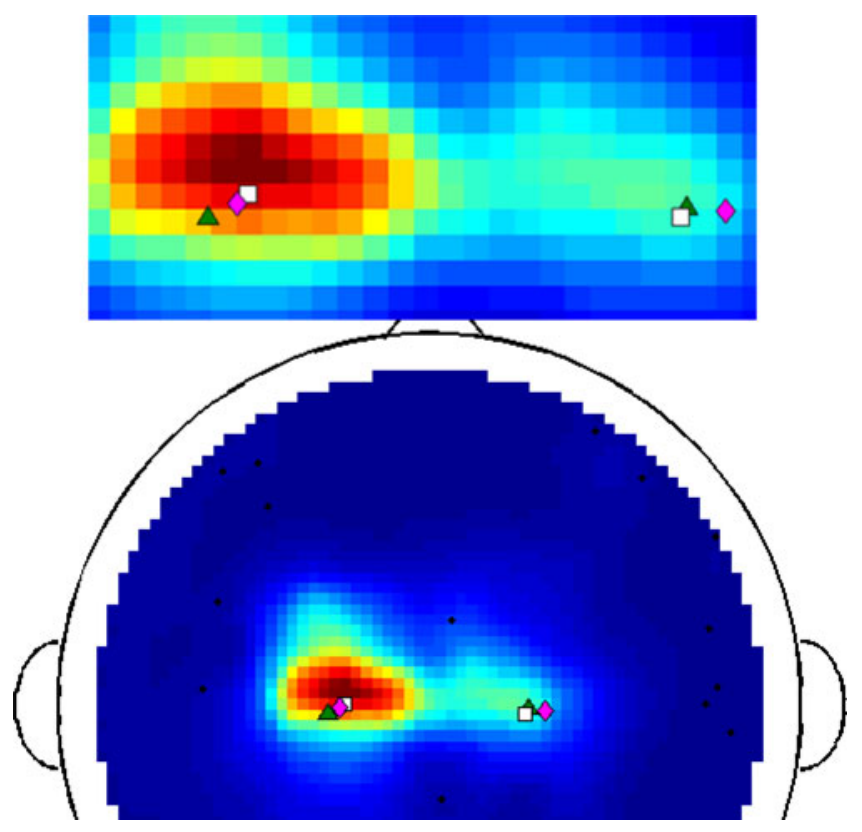

Fig. 2. Same as previous figure, but sources on the brain surface are more closely spaced, near sensors $\mathrm{C} 1$ and $\mathrm{C} 2$. Spatial spectrum is from MUSIC (LCMV gives similar results), NSF and DML estimates indicated by square and diamond as before. Green triangles denote the true source locations.

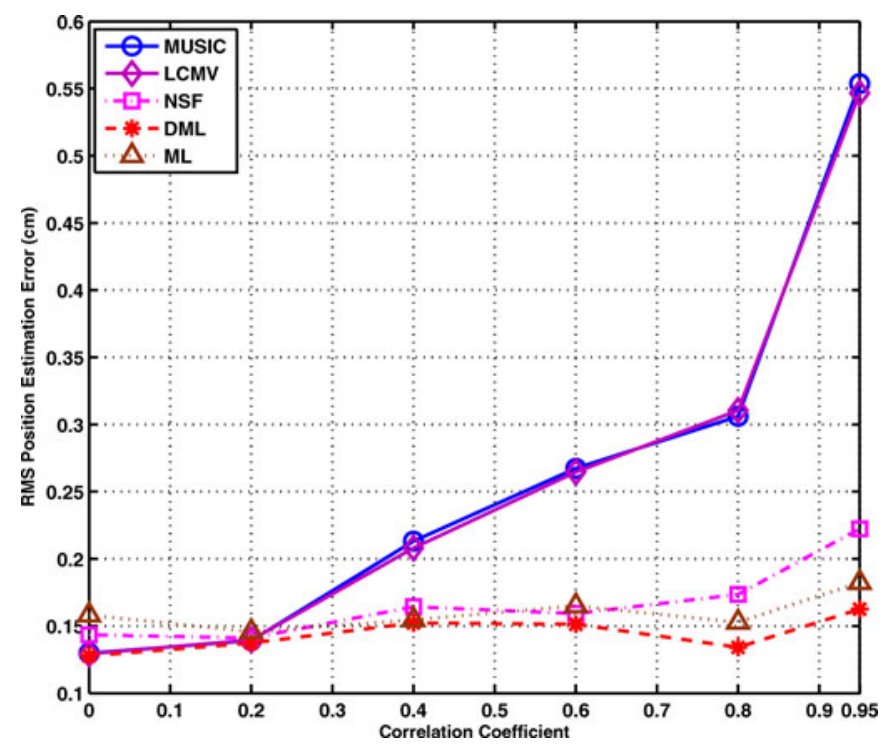

Fig. 3. RMS position estimation errors for MUSIC, LCMV, NSF, DML, and ML.

(not noise-canceling) headphones and the experiments were conducted in a standard office environment. The subject was not blindfolded. EEG signals were measured using two NeXus32 (MindMedia, Roermond-Herten, The Netherlands) systems with linked trigger ports and driven by a synchronization signal so that they were effectively acting as one system. Each NeXus32 system had a built-in low-pass filter with cutoff frequency at $138 \mathrm{~Hz}$. The stimulus consisted of a 1-kHz pure tone presented to the subject's left ear. Time intervals with no sound were used to generate control state data. The experiment was broken down into 1124 frames, each frame consisting of data recorded with 


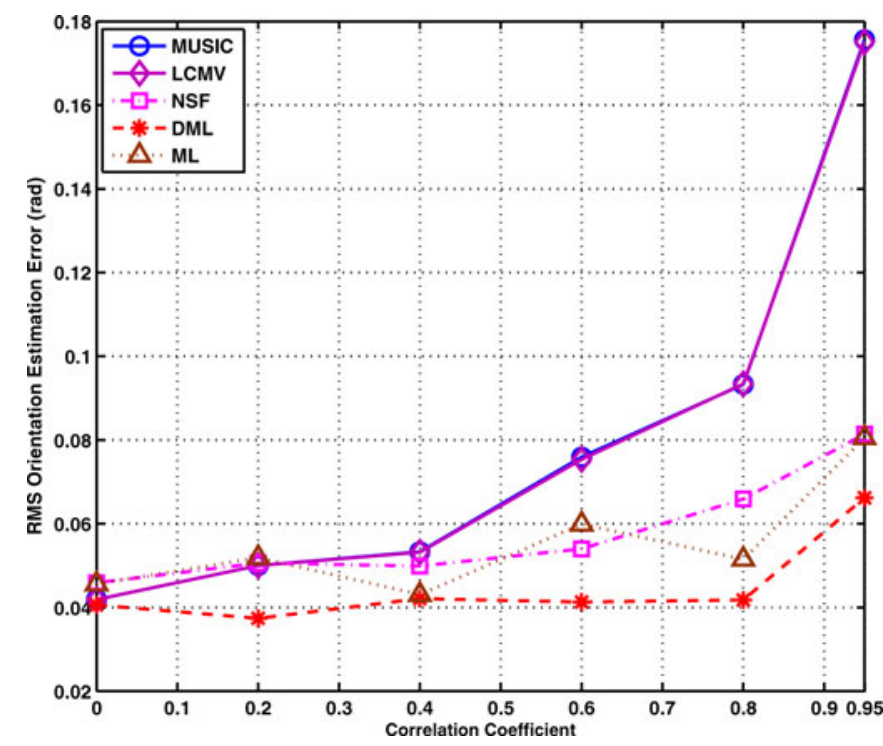

Fig. 4. RMS orientation estimation errors for MUSIC, LCMV, NSF, DML, and ML.

a 512-Hz sampling rate after each possible stimulus event. The length of each frame was randomly varied between $700-800 \mathrm{~ms}$. If the frame contained a stimulus, it occurred during the first 100 ms and was followed by silence to allow neural activity to die down before the next possible stimulus event. The content of each frame was randomly varied between the stimulus and silence. The outputs of 61 electrodes were recorded, similar to the setup assumed in the simulations of the previous section. Prior to application of the algorithms described earlier, the data were detrended, the first $700 \mathrm{~ms}$ of each frame was isolated, and then all frames of the same type were averaged together to create the control state and activity state data.

Auditory stimuli presented at one of the ears are conveyed through auditory pathways until they reach the brain. At the beginning, the inputs cross to the opposite side in the hindbrain and then there is a recrossing of information so that information from the ear reaches both hemispheres [30], [31]. Due to the mechanism described earlier, we might expect a correlated source scenario when the tone is applied to one of the ears of the subject. Furthermore, for auditory-evoked potentials, the P50 and N100 waves are the most relevant to our experiments. They have latencies of about 50 and $100 \mathrm{~ms}$ from stimulus onset and are reported to have a strong contribution from the primary auditory cortex [2], [11]. An SVD of the control state data matrix with size $(61 \times 358)$ revealed that about $99 \%$ of its energy was confined to a rank-18 subspace, and thus, the number of interferers was set to 18 . The number of sources-of-interest was set to 2 . For the activity state data, only samples between 25 to $158 \mathrm{~ms}$ (69 samples) after application of the stimulus were used for localization, in order to isolate the P50 and N100 ERP peaks. Before applying the algorithms, the NP technique was applied to the activity-state data for interference suppression.

Fig. 5 shows the spatial spectra generated by MUSIC and LCMV. Dipole location estimates obtained using NSF (white squares) and the DML method (magenta diamonds) are plotted
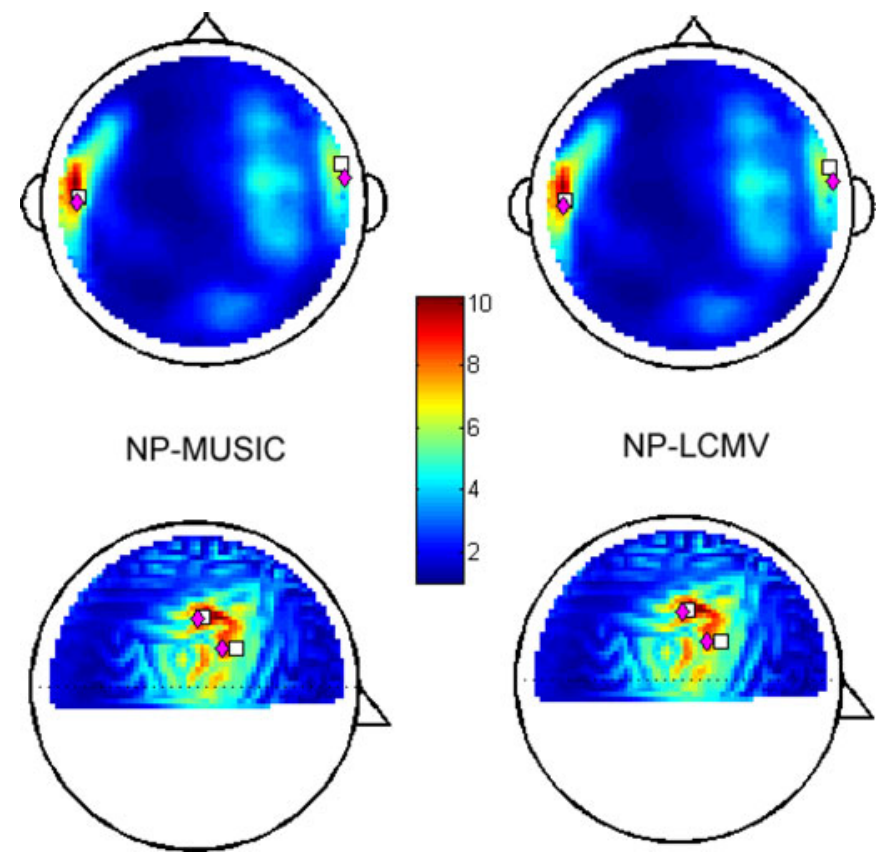

Fig. 5. Spatial spectra and location estimates obtained by discussed algorithms using experimental activity data. The white squares and magenta diamonds represent the position estimates obtained by NSF and UML, respectively.

as well. NSF and DML provide location estimates that are consistent with sources located on the auditory cortex. On the other hand, the energy in the MUSIC and LCMV spectra is significantly smeared, and the source on the right side is very weak and the corresponding estimate is not well defined (this is similar to what is observed in Fig. 1). The correlation coefficient $\rho_{\mathrm{s}_{1}, \mathrm{~s}_{2}}$ between the two estimated sources was calculated using

$$
\rho_{\mathbf{s}_{1}, \mathbf{s}_{2}}=\frac{1}{n_{A}-1} \sum_{i=1}^{n_{A}}\left(\frac{s_{1}(i)-\overline{\mathbf{s}_{1}}}{\sigma_{\mathbf{s}_{1}}}\right)\left(\frac{s_{2}(i)-\overline{\mathbf{s}_{2}}}{\sigma_{\mathbf{s}_{2}}}\right)
$$

where $\overline{\mathbf{s}_{i}}$ and $\sigma_{\mathbf{s}_{i}}$ are the mean and standard deviation of $\mathbf{s}_{i}$. The correlation coefficients between the two sources identified by NSF and DML are 0.8820 and 0.8989 , respectively, indicating that indeed this corresponds to a situation with high correlation.

\section{CONCLUSION}

We have presented two efficient algorithms for handling EEG source localization problems involving highly correlated signals, which can lead to difficulties when standard highresolution approaches such as MUSIC or LCMV are applied. The two algorithms, referred to as DML and NSF, require a less complex optimization than standard ML, but both will have asymptotically the same performance as ML. The estimation accuracy of DML and NSF was verified through simulations that showed its increasing advantage over MUSIC and LCMV with increasing signal correlation. A real-data experiment with auditory stimuli applied to both ears of a human subject was conducted to create a scenario with highly correlated activity data. The results indicate that DML and NSF provide results consistent with that expected in this case, with location estimates concentrated near the auditory cortex. 


\section{REFERENCES}

[1] Y. S. Chen, C. Y. Cheng, J. C. Hsieh, and L. F. Chen, "Maximum contrast beamformer for electromagnetic mapping of brain activity," IEEE Trans. Biomed. Eng., vol. 53, no. 9, pp. 1765-1774, Sep. 2006.

[2] K. Sekihara, K. Hild, and S. Nagarajan, "A novel adaptive beamformer for MEG source reconstruction effective when large background brain activities exist," IEEE Trans. Biomed. Eng., vol. 53, no. 9, pp. 17551764, Sep. 2006.

[3] H. B. Hui, D. Pantazis, S. L. Bressler, and R. M. Leahy, "Identifying true cortical interactions in MEG using the nulling beamformer," NeuroImage, vol. 49, pp. 3161-3174, 2010.

[4] M. Diwakar, M.-X. Huang, R. Srinivasan, D. L. Harrington, A. Robb, A. Angeles, L. Muzzatti, R. Pakdaman, T. Song, R. J. Theilmann, and R. R. Lee, "Dual-core beamformer for obtaining highly correlated neuronal networks in MEG," NeuroImage, vol. 54, pp. 253-263, 2011.

[5] J. C. Mosher, P. S. Lewis, and R. M. Leahy, "Multiple dipole modeling and localization from spatio-temporal MEG data," IEEE Trans. Biomed. Eng., vol. 39, no. 6, pp. 541-557, Jun. 1992.

[6] R. Schmidt, "Multiple emitter location and signal parameter estimation," IEEE Trans. Antenn. Propag., vol. 34, no. 3, pp. 276-280, Mar. 1986.

[7] B. D. van Veen, W. van Drongelen, M. Yuchtman, and A. Suzuki, "Localization of brain electrical activity via linearly constrained minimum variance spatial filtering," IEEE Trans. Biomed. Eng., vol. 44, no. 9, pp. 867-880, Sep. 1997.

[8] M. Sun, "An efficient algorithm for computing multi-shell spherical volume conductor models in EEG dipole source localization," IEEE Trans. Biomed. Eng., vol. 44, no. 12, pp. 1243-1252, Dec. 1997.

[9] J. Sarvas, "Basic mathematical and electromagnetic concepts of the biomagnetic inverse problem," Phys. Med. Biol., vol. 32, pp. 11-22, 1987.

[10] K. Sekihara, S. S. Nagarajan, D. Poeppel, A. Marantz, and Y. Miyashita, "Application of an MEG eigenspace beamformer to reconstructing spatiotemporal activities of neural sources," Hum. Brain Mapp., vol. 15, pp. 199-215, 2002.

[11] S. S. Dalal, K. Sekihara, and S. S. Nagarajan, "Modified beamformers for coherent source region suppression," IEEE Trans. Biomed. Eng., vol. 53, no. 7, pp. 1357-1363, Jul. 2006.

[12] J. C. Mosher and R. M. Leahy, "Source localization using recursively applied and projected (RAP) MUSIC," IEEE Trans. Signal Process., vol. 47, no. 2, pp. 332-340, Feb. 1999.

[13] X.-L. Xu, B. Xu, and B. He, "An alternative subspace approach to EEG dipole source localization," Phys. Med. Biol., vol. 49, pp. 327-343, 2004.

[14] B. Lütkenhöner, "Dipole source localization by means of maximum likelihood estimation: Theory and simulations," Electroencephalogr.Clin. Neurophysiol., vol. 106, pp. 314-321, 1998.

[15] S. Baillet, J. C. Mosher, and R. M. Leahy, "Electromagnetic brain mapping," IEEE Signal Process. Mag., vol. 18, no. 6, pp. 14-30, Nov. 2001.

[16] A. Dogandžić, and A. Nehorai, "Estimating evoked dipole responses in unknown spatially correlated noise with EEG/MEG arrays," IEEE Trans. Signal Process., vol. 48, no. 1, pp. 13-25, Jan. 2000.

[17] J. C. de Munck, H. M. Huizenga, L. J. Waldorp, and R. A. Heethaar, "Estimating stationary dipoles from MEG/EEG data contaminated with spatially and temporally correlated background noise," IEEE Trans. Signal Process., vol. 50, no. 7, pp. 1565-1572, Jul. 2002.

[18] J. C. de Munck, "The estimation of time varying dipoles on the basis of evoked potentials," Electroencephalogr. Clin. Neurophysiol., vol. 77, pp. 156-160, 1990.

[19] A. Swindlehurst and M. Viberg, "Subspace fitting with diversely polarized antenna arrays," IEEE Trans. Antenn. Propag., vol. 41, no. 12, pp. 16871694, Dec. 1993

[20] J. C. de Munck and M. J. Peters, "A fast method to compute the potential in the multi sphere model," IEEE Trans. Biomed. Eng., vol. 40, no. 11, pp. 1166-1174, Nov. 1993.

[21] B. N. Cuffin, "EEG localization accuracy improvements using realistically shaped head models," IEEE Trans. Biomed. Eng., vol. 43, no. 3, pp. 299303, Mar. 1996.

[22] K. Sekihara, S. S. Nagarajan, D. Poeppel, and A. Marantz, "Performance of an MEG adaptive-beamformer technique in the presence of correlated neural activities: Correlation-coefficient estimation and time-course retrieval," IEEE Trans. Biomed. Eng., vol. 49, no. 12, pp. 1534-1546, Dec. 2002.

[23] J. J. Ermer, J. C. Mosher, M. Huang, and R. M. Leahy, "Paired MEG data set source localization using recursively applied and projected (RAP) MUSIC," IEEE Trans. Biomed. Eng., vol. 47, no. 9, pp. 1248-1260, Sep. 2000
[24] S. C. Wu, A. L. Swindlehurst, P. T. Wang, and Z. Nenadic, "Projection vs. prewhitening for EEG interference suppression," IEEE Trans. Biomed. Eng., vol. 59, no. 5, pp. 1329-1338, May 2012.

[25] P. Stoica and K. Sharman, "Maximum likelihood methods for direction-ofarrival estimation," IEEE Trans. Acoust. Speech, Signal Process., vol. 38 , no. 7, pp. 1132-1143, Jul. 1990.

[26] R. Oostenveld and P. Praamstra, "The five percent electrode system for high-resolution EEG and ERP measurements," Clin. Neurophysiol., vol. 112, pp. 713-719, 2001

[27] B. N. Cuffin and D. Cohen, "Comparison of the magnetoencephalogram and electroencephalogram," Electroencephalogr. Clin. Neurophysiol., vol. 47, pp. 132-146, 1979

[28] S. J. Luck, An Introduction to the Event-Related Potential Technique. Cambridge, MA: MIT Press, 2005.

[29] V. Reddy, A. Paulraj, and T. Kailath, "Performance analysis of the optimum beamformer in the presence of correlated sources and its behavior under spatial smoothing," IEEE Trans. Acoust. Speech, Signal Process., vol. 35, no. 7, pp. 927-936, Jul. 1987.

[30] E. Pekkonen, M. Huotilainen, J. Virtanen, J. Sinkkonen, T. Rinne, R. J. Ilmoniemi, and R. Näätänen, "Age-related functional differences between auditory cortices: A whole-head MEG study," NeuroReport, vol. 6, pp. 1803-1806, 1995.

[31] B. Kolb and I. Q. Whishaw, Introduction to Brain and Behavior. Worth, 2001.

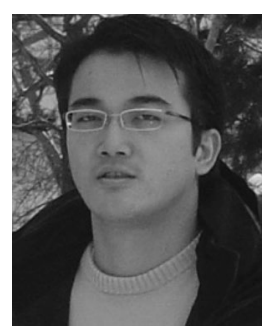

Shun Chi Wu (S'09) received the B.S. and M.S. degrees in engineering and system science both from the National Tsing Hua University, Hsinchu, Taiwan, in 2000 and 2002, respectively. He is currently working toward the Ph.D. degree in the Department of Electrical Engineering and Computer Science, University of California, Irvine.

From 2003 to 2007, he was a Research Assistant at National Space Organization, Hsinchu, Taiwan. His research interests include interference mitigation, feature extraction, source localization/reconstruction, and brain connectivity analysis.

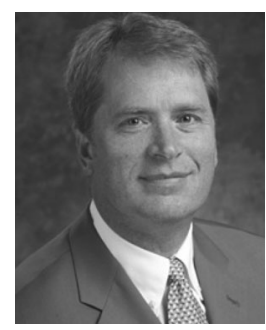

A. Lee Swindlehurst (S'83-M'84-SM'99-F'04) received the B.S. (summa cum laude) and M.S. degrees in electrical engineering both from Brigham Young University, Provo, Utah, in 1985 and 1986, respectively, and the $\mathrm{Ph} . \mathrm{D}$. degree in electrical engineering from Stanford University, Stanford, CA, in 1991.

From 1986 to 1990, he was employed at the ESL, Inc., of Sunnyvale, CA, where he was involved in the design of algorithms and architectures for several radar and sonar signal processing systems. From 1990 to 2007, he with the Faculty of the Department of Electrical and Computer Engineering, Brigham Young University, where he was a Full Professor and served as the Department Chair from 2003 to 2006 During 1996-1997, he held a joint appointment as a Visiting Scholar at both Uppsala University, Uppsala, Sweden, and at the Royal Institute of Technology, Stockholm, Sweden. From 2006 to 2007, he was on leave working as the VicePresident of Research for ArrayComm LLC in San Jose, CA. He is currently a Professor in the Department of Electrical Engineering and Computer Science, University of California, Irvine. His research interests include sensor array signal processing for radar and wireless communications, detection and estimation theory, and system identification, and he has more than 220 publications in these areas.

Dr. Swindlehurst is a past Secretary of the IEEE Signal Processing Society, past Editor-in-Chief of the IEEE JOURNAL OF SELECTED TOPICS IN SIGNAL PROCESSING, and past member of the Editorial Boards for the EURASIP Journal on Wireless Communications and Networking, IEEE Signal Processing Magazine, and the IEEE TRANSACTIONS ON Signal PROCESSING. He is a recipient of several paper awards: the 2000 IEEE W. R. G. Baker Prize Paper Award, the 2006 and 2010 IEEE Signal Processing Society's Best Paper Award, the 2006 IEEE Communications Society Stephen O. Rice Prize in the Field of Communication Theory, and is co-author of a paper that received the IEEE Signal Processing Society Young Author Best Paper Award in 2001 


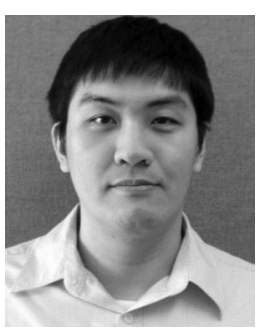

Po T. Wang (S'09) received the B.S. degree in bioengineering from Rice University, Houston, TX, in 2007, and the M.S. degree in biomedical engineering from the University of California, Irvine, in 2008, where he is currently working toward the Ph.D. degree in the Department of Biomedical Engineering.

His research interests include brain-computer interfaces and machine learning.

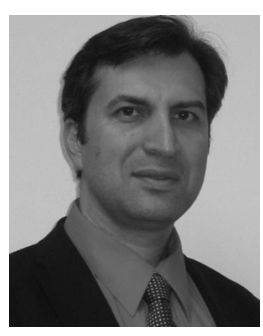

Zoran Nenadic (M'06) received the Diploma degree in control engineering from the University of Belgrade, Belgrade, Serbia, in 1995, and the M.S and D.Sc. degrees in systems science and mathematics both from Washington University, St. Louis, MO, in 1998 and 2001, respectively.

From 2001 to 2005, he was a Postdoctoral Scholar with the Division of Engineering and Applied Science at the California Institute of Technology, Pasadena. Since 2005, he has been with the Department of Biomedical Engineering, University of California, Irvine, where he is currently an Associate Professor. His research interest include the area of neuroengineering, biomedical signal processing and pattern recognition, biological neural network modeling, and biomedical devices and sensors.

Dr. Nenadic is the recipient of the National Science Foundation Faculty Early Career Development Award. He is a member of the Mathematical Association of America and the Society for Neuroscience. 